\title{
Desipramine improves upper airway collapsibility and reduces OSA severity in patients with minimal muscle compensation
}

\author{
Luigi Taranto-Montemurro ${ }^{1}$, Scott A. Sands ${ }^{1,2}$, Bradley A. Edwards ${ }^{1,3,4}$, \\ Ali Azarbarzin ${ }^{1}$, Melania Marques ${ }^{1}$, Camila de Melo $^{1}$, Danny J. Eckert ${ }^{5}$, \\ David P. White ${ }^{1}$ and Andrew Wellman ${ }^{1}$
}

\begin{abstract}
Affiliations: ${ }^{1}$ Division of Sleep and Circadian Disorders, Depts of Medicine and Neurology, Brigham \& Women's Hospital \& Harvard Medical School, Boston, MA, USA. ${ }^{2}$ Dept of Allergy, Immunology and Respiratory Medicine and Central Clinical School, The Alfred and Monash University, Melbourne, Australia. ${ }^{3}$ Sleep and Circadian Medicine Laboratory, Department of Physiology, Monash University, Melbourne, Australia. ${ }^{4}$ School of Psychological Sciences and Monash Institute of Cognitive and Clinical Neurosciences, Monash University, Melbourne, Australia. ${ }^{5}$ Neuroscience Research Australia (NeuRA) and the University of New South Wales, Randwick, Australia.
\end{abstract}

Correspondence: Luigi Taranto-Montemurro, 221 Longwood Ave. Suite BLI038A, Boston, MA 02115, USA. E-mail: taranto.luigidagmail.com

ABSTRACT We recently demonstrated that desipramine reduces the sleep-related loss of upper airway dilator muscle activity and reduces pharyngeal collapsibility in healthy humans without obstructive sleep apnoea (OSA). The aim of the present physiological study was to determine the effects of desipramine on upper airway collapsibility and apnoea-hypopnea index (AHI) in OSA patients.

A placebo-controlled, double-blind, randomised crossover trial in 14 OSA patients was performed. Participants received treatment or placebo in randomised order before sleep. Pharyngeal collapsibility (critical collapsing pressure of the upper airway $\left.\left(P_{\text {crit }}\right)\right)$ and ventilation under both passive $\left(V^{\prime} 0\right.$,passive) and active $\left(V^{\prime} 0\right.$,active $)$ upper airway muscle conditions were evaluated with continuous positive airway pressure (CPAP) manipulation. AHI was quantified off CPAP.

Desipramine reduced active $P$ crit (median (interquartile range) $-5.2(4.3) \mathrm{cmH}_{2} \mathrm{O}$ on desipramine versus -1.9 (2.7) $\mathrm{cmH}_{2} \mathrm{O}$ on placebo; $\left.\mathrm{p}=0.049\right)$ but not passive Pcrit $\left(-2.2\right.$ (3.4) versus -0.7 (2.1) $\left.\mathrm{cmH}_{2} \mathrm{O} ; \mathrm{p}=0.135\right)$. A greater reduction in AHI occurred in those with minimal muscle compensation (defined as $V^{\prime} 0$, active $-V^{\prime} 0$, passive) on placebo $(\mathrm{r}=0.71, \mathrm{p}=0.009)$. The reduction in AHI was driven by the improvement in muscle compensation $(\mathrm{r}=0.72, \mathrm{p}=0.009)$.

In OSA patients, noradrenergic stimulation with desipramine improves pharyngeal collapsibility and may be an effective treatment in patients with minimal upper airway muscle compensation.

@ERSpublications

Desipramine administered before sleep reduces pharyngeal collapsibility in patients with obstructive sleep apnoea http://ow.ly/1auA302CL8F

\begin{abstract}
This article has supplementary material available from erj.ersjournals.com
Received: April 262016 | Accepted after revision: July 192016 | First published online: Oct 062016

Support statement: This research project received generous philanthropic funding from Fan Hongbing, President of OMPA Corporation, Kaifeng, China. This work was also supported by the National Institutes of Health grants R01 HL102321 and P01 HL095491 as well as the Harvard Catalyst Clinical Research Center: UL1 RR 025758-01. L. Taranto-Montemurro is supported by the American Heart Association (15POST25480003). B.A. Edwards is supported by the National Health and Medical Research Council (NHMRC) of Australia's CJ Martin Overseas Biomedical Fellowship (1035115). S.A. Sands was supported by the NHMRC of Australia (1053201), the Menzies Foundation, the American Heart Association (15SDG25890059) and the American Thoracic Society Foundation, and was also co-investigator on grants from the NHMRC (1064163) and NIH (R01 HL128658). M. Marques is supported by Capes Foundation, Ministry of Education of Brazil. D.J. Eckert is supported by a NHMRC R.D. Wright Fellowship (1049814). Funding information for this article has been deposited with the Open Funder Registry.
\end{abstract}

Conflict of interest: Disclosures can be found alongside this article at erj.ersjournals.com

Copyright OERS 2016 


\section{Introduction}

Obstructive sleep apnoea (OSA) is a state-dependent disease which occurs in at least 5-10\% [1] of the general population. People with OSA can breathe normally during wakefulness despite having a narrow, compliant upper airway. During sleep, the withdrawal of drive to upper airway dilator muscles leads to the collapse of pharyngeal structures with subsequent episodes of reduced ventilation plus intermittent hypoxia and hypercapnia [2].

Continuous positive airway pressure (CPAP) is still the primary treatment for most patients. While CPAP is virtually always effective (as it mechanically splints the airway open), approximately $25 \%$ of patients refuse CPAP or discontinue it in the first 2 weeks. Furthermore, for those who continue for more than 1 month, adequate adherence can vary between $46 \%$ and $89 \%$ depending on definitions of adherence and length of follow-up [3]. The low adherence to treatment is a major problem, as untreated OSA increases morbidity and mortality [4-6].

While current evidence has demonstrated that OSA is caused by multiple factors including a highly collapsible upper airway; clearly, there are also some individuals with OSA who have little or no ability to activate their upper airway muscles during sleep (poor neuromuscular compensation) [7-9]. In these patients, a key cause of OSA is the withdrawal of neural drive to upper airway dilator muscles during sleep with inadequate neuromuscular compensation which leads to the collapse of pharyngeal structures.

Interestingly, animal studies [10] support the notion that the progressive withdrawal of noradrenergic tone, from wakefulness to sleep, is the major mechanism causing non-rapid eye movement (NREM) sleeprelated pharyngeal hypotonia. Consistent with findings in animal research, we recently found that desipramine, a tricyclic antidepressant (TCA) that inhibits the norepinephrine reuptake receptor in the central nervous system with the highest affinity in this pharmaceutical class, reduces the sleep-related loss of genioglossus activity and improves pharyngeal collapsibility in healthy humans without OSA [11].

However, it is not known whether desipramine similarly reduces the sleep-related loss of muscle activity and improves the pharyngeal patency in OSA patients. The effect of another TCA (protriptyline) on OSA severity was previously tested in observational studies [12-14] and small randomised controlled trials $[15,16]$ with inconsistent results.

The primary goal of this study was to assess the effect of desipramine on pharyngeal collapsibility in OSA (critical collapsing pressure of the upper airway $\left.\left(P_{\text {crit }}\right)\right)$. As a secondary outcome, we also assessed the impact of desipramine on OSA severity (apnoea-hypopnea index (AHI)) quantified on a portion of the night without CPAP. We hypothesised that desipramine would effectively reduce OSA severity in patients with mild-to-moderate upper-airway collapsibility and poor upper airway muscle compensation.

\section{Methods}

\section{Participants}

Patients with OSA (AHI>15 events $\cdot \mathrm{h}^{-1}$ sleep) aged 18-65 years were included in the study protocol. Individuals were excluded if they were taking medications known to influence breathing in sleep or wake states (i.e. hypnotics, antipsychotics or anxiolytics), and psychostimulants, or muscle physiology (i.e. myorelaxant). All patients were required to abstain from consuming alcoholic beverages $24 \mathrm{~h}$ before the study. Also excluded were subjects with benign prostatic hyperplasia or urinary retention, which can be exacerbated by TCAs or individuals taking psychiatric medications. The protocol was approved by the Partners Institutional Review Board at Brigham and Women's Hospital. All subjects provided written informed consent prior to enrolment in the study.

\section{Protocol}

Two overnight sleep studies were performed 1 week apart: a placebo night and a desipramine (200 mg by mouth) night, in a double-blind, randomised, placebo-controlled design (clinical trial NCT02436031). Study medications were prepared by Brigham and Women's Hospital Investigational Drug Service (IDS) and were placed in identical capsules that could not be identified by study personnel/participants. Randomisation was performed by the IDS; all data analyses and subject exclusions were performed before unblinding of the intervention allocation. For each night, the subjects arrived at the sleep laboratory at approximately 19:00 $\mathrm{h}$. Placebo or desipramine was administered approximately $2 \mathrm{~h}$ before lights out. Time of lights out was established according to the patients' habitual schedule and kept constant between the two study nights. Once the patient had been set up for overnight monitoring, the following procedures were performed.

\section{Measurements and equipment}

Anthropometric data were collected on both study nights. Circumference of the neck was measured at the superior border of the cricothyroid cartilage and waist circumference was measured at the highest point of the iliac crest. 
In addition to the standard clinical montage for polysomnography [17], subjects breathed through a sealed nasal mask attached to a pneumotachometer (Hans-Rudolph, Kansas City, MO, USA) and a pressure transducer (Validyne, Northridge, CA, USA) in order to measure airflow and mask pressure respectively. Epiglottic pressure $(P$ epi) was determined with a small, flexible pressure-tipped catheter (Millar Instruments, Houston, TX, USA) that was inserted through a decongested (oxymetazoline- $\mathrm{HCl}$ ) and anaesthetised $(4 \%$ lidocaine) nostril until the tip of the catheter was located $1-2 \mathrm{~cm}$ caudal to the base of the tongue. The catheter was then securely taped in place after visual inspection through the mouth.

EMG activity from the genioglossus (EMGGG) muscle was measured using two unipolar intramuscular electrodes to create a bipolar recording as described previously $[8,11,18]$.

\section{Sleep in supine position off CPAP}

At least $3 \mathrm{~h}$ of data were collected in the supine position for calculation of the AHI. This data collection occurred before and after upper airway physiology and the phenotype traits were quantified.

\section{Upper airway physiology using CPAP manipulations during NREM}

Subjects were asked to sleep supine and were connected to a positive/negative pressure source (Philips-Respironics, Murrysville, PA, USA) to enable rapid switching between pressure levels. When stable sleep was reached, the pressure in the mask was increased to the required level to abolish airflow limitation, as determined by the airflow waveform and epiglottic pressure signals. Following a baseline recording period of $5 \mathrm{~min}$, the CPAP level was reduced to varying suboptimal pressures using two approaches (figure 1): 1) CPAP was lowered acutely to subtherapeutic levels to assess the upper airway collapsibility under eupnoeic (i.e. "passive") conditions; and 2) $\mathrm{CPAP}$ was lowered gradually $\left(<1 \mathrm{cmH}_{2} \mathrm{O}\right.$ per min) to reduce ventilation and thereby raise ventilatory drive and pharyngeal muscle activity. During this procedure we assessed the EMGGG response to progressively-greater negative epiglottic pressure swings (genioglossus muscle responsiveness) and upper airway collapsibility under maximally-active conditions without cortical arousal.

After approximately $2 \mathrm{~h}$ of upper airway physiology measurements, CPAP was removed and subjects returned to spontaneous breathing in the supine position to collect additional data for AHI calculation at atmospheric pressure. As much data were recorded from rapid eye movement (REM) and NREM sleep as possible.

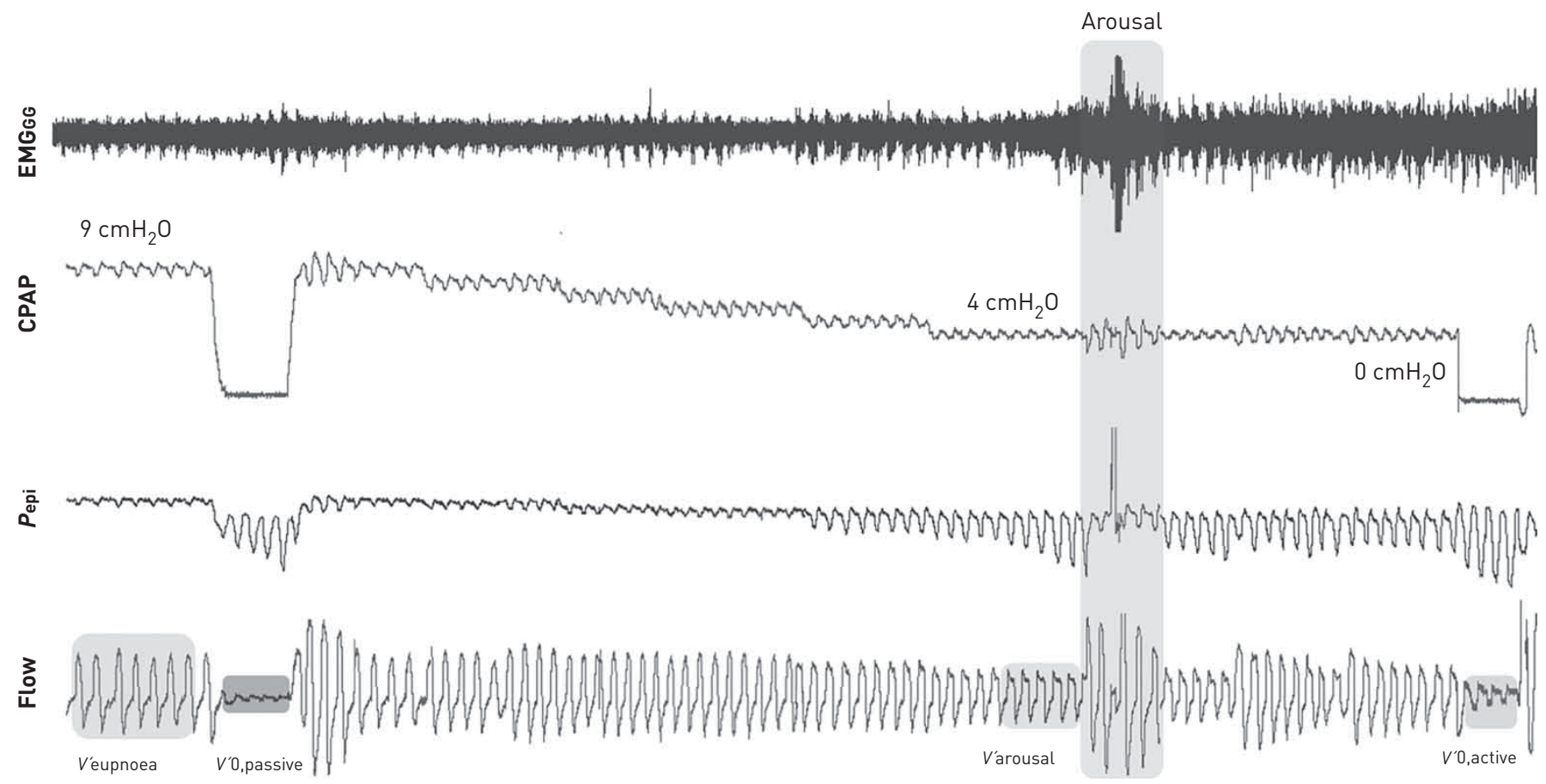

FIGURE 1 Determination of the four ventilatory parameters. When stable sleep is reached at a continuous positive airway pressure (CPAP) level that abolishes flow limitation $\left(9 \mathrm{cmH}_{2} \mathrm{O}\right.$ in this example), ventilation on optimum CPAP ( $V^{\prime}$ eupnoea) is determined by averaging several minutes of ventilation during stable breathing. CPAP is then lowered to $0 \mathrm{cmH}_{2} \mathrm{O}$ acutely (passive drop) to assess ventilation under eupnoeic conditions ( $V^{\prime} 0$, passive). Subsequently, the CPAP is lowered gradually $\left(<1 \mathrm{cmH}_{2} \mathrm{O}\right.$ per min) to slowly reduce ventilation and thereby raise ventilatory drive, increase epiglottic pressure ( $P$ epil swings and pharyngeal muscle activity (represented here by electromyography of genioglossus (EMGGG)]. During this procedure we also assessed the average ventilation of the five stable breaths preceding the arousal if they are flow-limited ( $V^{\prime}$ arousal), and the ventilation at atmospheric pressure under maximally active conditions ( $V^{\prime} 0$,active). 


\section{Data analysis}

The raw EMGGG was processed and quantified as \% maximum (\%max) as previously described [19]. Apnoeas and hypopneas were scored using standard American Academy of Sleep Medicine guidelines [20], and the arousal index and AHI values refer to the supine sleep periods without CPAP.

As described in previous studies [11, 19], for each subject we also measured the following. 1) Genioglossus responsiveness to negative pharyngeal pressure: the increase in peak EMGGG (\%max) per unit change $\left(\mathrm{cmH}_{2} \mathrm{O}\right)$ in epiglottic pressure during each gradual pressure drop. 2) Passive upper airway collapsibility: this was quantified by passive $P_{\text {crit, }}$ the $\mathrm{x}$-intercept of the regression line fitted to the peak inspiratory flow versus mask pressure obtained during abrupt CPAP drops (third to fifth flow-limited breaths). 3) Active upper airway collapsibility: we calculated the critical pressure yielding zero flow when the upper airway

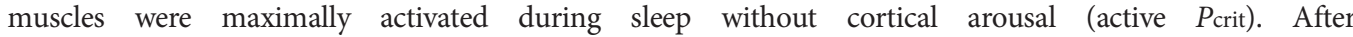
maximally-raising pharyngeal muscle activity (via gradual CPAP reduction), CPAP was switched acutely to various lower CPAP levels (including $0 \mathrm{cmH}_{2} \mathrm{O}$ ), and the first breath was assessed if flow-limited. Similar to passive $P_{\text {crit, }}$ the active $P_{\text {crit }}$ was taken as the $\mathrm{x}$-intercept of the regression line fitted to flow-pressure data. 4) Arousal threshold: the arousal threshold was quantified as the mean of the nadir negative epiglottic pressure immediately preceding an arousal at the end of an obstructive apnoea or hypopnea.

\section{Ventilatory parameters}

As previously described [21], we also quantified the ventilation on optimum CPAP ( $V^{\prime}$ eupnoea), ventilation at a nasal pressure of $0 \mathrm{cmH}_{2} \mathrm{O}$ when the pharyngeal muscles were relatively passive ( $V^{\prime}$, passive) or maximally active $\left(V^{\prime}\right.$,active) and the minimum tolerable ventilation leading to an arousal ( $V^{\prime}$ arousal $)$. Muscle compensation was calculated as the improvement in ventilation due to increased compensatory upper airway muscle activity $\left(V^{\prime} 0\right.$,active $-V^{\prime} 0$,passive $)$.

\section{Dynamic loop gain}

Loop gain quantifies the magnitude of the ventilatory "response" that follows a ventilatory "disturbance" (reduced ventilation with apnoea/hypopnoea). The magnitude of loop gain (response/disturbance), which represents the sensitivity of the ventilatory control system was calculated from polysomnographic flow signals as previously described [22] as the response to a 1-cycle. $\mathrm{min}^{-1}$ disturbance; which was calculated from the portions of the study off CPAP, the same parts used to calculate AHI.

\section{Statistical analysis}

Based on previous data collected in healthy subjects, the current study was powered to detect a reduction in the passive $P_{\text {crit }}$ by $1.2 \pm 1.5 \mathrm{cmH}_{2} \mathrm{O}$ on desipramine compared to placebo (80\% power and a $5 \%$ level of significance). Variables were compared using a Wilcoxon matched-pairs signed rank test for paired data and Mann-Whitney test for unpaired data, with a $\mathrm{p}$-value $<0.05$ considered statistically significant. Data are presented as a median (interquartile range). Statistical analyses were performed using Graph Pad Prism 6.0 (Graphpad Software, La Jolla, CA, USA).

\section{Results}

\section{Participants}

A total of 16 patients were enrolled in the study. One patient did not tolerate the study setup and another was excluded because of insufficient sleep time. Therefore, 14 subjects were analysed for upper airway physiology on both nights. The characteristics of these patients are described in table 1 . None of the patients were current smokers. One patient experienced mild dizziness in the morning after desipramine administration. The symptom lasted for a few hours and the patient spontaneously recovered.

One subject could not sleep supine on the placebo night and, thus, AHI could not be calculated. However, we asked this subject to come back for an additional night for a standard polysomnography without drug to calculate AHI during supine NREM sleep. This value was only used for post hoc exploratory analysis (relationships of change in AHI versus other variables). Two subjects without moderate-to-severe OSA (AHI $<15$ events. $h^{-1}$ ) were excluded from the post hoc exploratory analysis (see online data supplement for further explanation).

\section{Effects on upper airway collapsibility}

Passive collapsibility was unchanged between nights while active $P_{\text {crit }}$ was significantly lower (i.e. less collapsible airway) during the desipramine night compared to placebo (median change -3.1 (4.8)) (figure 2). A post hoc analysis revealed that during active $P_{\text {crit }}$ drops, phasic EMGGG activity was higher on desipramine (see online data supplement), indicating that desipramine increased upper airway stiffness by enhancing phasic activity of the pharyngeal muscles. 
TABLE 1 Characteristics of the 14 patients analysed

$\begin{array}{lc}\text { Female } & 4(29 \%) \\ \text { Age years } & 55(13) \\ \text { BMI } \mathbf{~ g g} \cdot \mathbf{m}^{-2} & 31.1(4.0) \\ \text { Neck circumference cm } & 41.8(6.1) \\ \text { Waist circumference cm } & 112.5(12.3) \\ \text { OSA treatment } & \\ \text { CPAP } & 4(29 \%) \\ \text { Oral appliance } & 2(14 \%) \\ \text { Comorbidities } & \\ \text { Hypertension } & 5(35 \%) \\ \text { Diabetes } & 2(14 \%) \\ \text { Hypercholesterolaemia } & 2(14 \%) \\ \text { Rheumatoid arthritis } & 1(7 \%) \\ \text { Medications } & \\ \text { ACE-IARB } & 4(29 \%) \\ \text { Diuretic } & 1(7 \%) \\ \text { Beta blockers } & 1(7 \%) \\ \text { Ca }{ }^{++} \text {channel antagonist } & 2(14 \%) \\ \text { Antilipidaemic } & 2(14 \%) \\ \text { Oral hypoglycaemic } & 1(7 \%) \\ \text { NSAlDs } & 1(7 \%)\end{array}$

Data are presented as or median (interquartile range), unless otherwise stated. BMI: body mass index; OSA: obstructive sleep apnoea; CPAP: continuous positive airway pressure; ACE-I: angiotensin-converting enzyme inhibitor; ARB: angiotensin receptor blocker; NSAIDs: non-steroidal anti-inflammatory drugs.

\section{Effects on AHI, ventilatory traits and loop gain}

Group data of NREM AHI, arousal index and oxygen desaturation index during NREM sleep are shown in table 2. REM sleep off CPAP was recorded in both nights only for two subjects and we did not consider it for the comparative analysis. NREM AHI and oxygen desaturation index, were unchanged between nights as a group, while nadir arterial oxygen saturation was significantly higher on desipramine compared with placebo. Individual data describing the change in the NREM AHI are available in figure 3 and in the online supplement.

$V^{\prime}$ eupnoea, $V^{\prime}$ arousal, $V^{\prime} 0$,active and $V^{\prime} 0$,passive were calculated during CPAP dial down (figure 1 ) for 14 subjects and are shown in table 3 . There was no significant difference in $V^{\prime} 0$,active, muscle compensation ( $V^{\prime} 0$,active $-V$ '0,passive) or any other ventilatory traits on desipramine compared with placebo. However, desipramine increased $V^{\prime} 0$,passive, $V^{\prime} 0$,active or both in 10 out of 14 subjects: in five subjects this was associated with a reduction in AHI, with the abolition of OSA in two of these subjects. Additionally, three subjects had a worsening in $V^{\prime}$,passive and $V^{\prime}$,active associated with an increase in NREM AHI. Loop gain was not different on desipramine night compared with placebo night (0.60 (0.20) versus $0.61(0.24) ; \mathrm{p}>0.5)$.
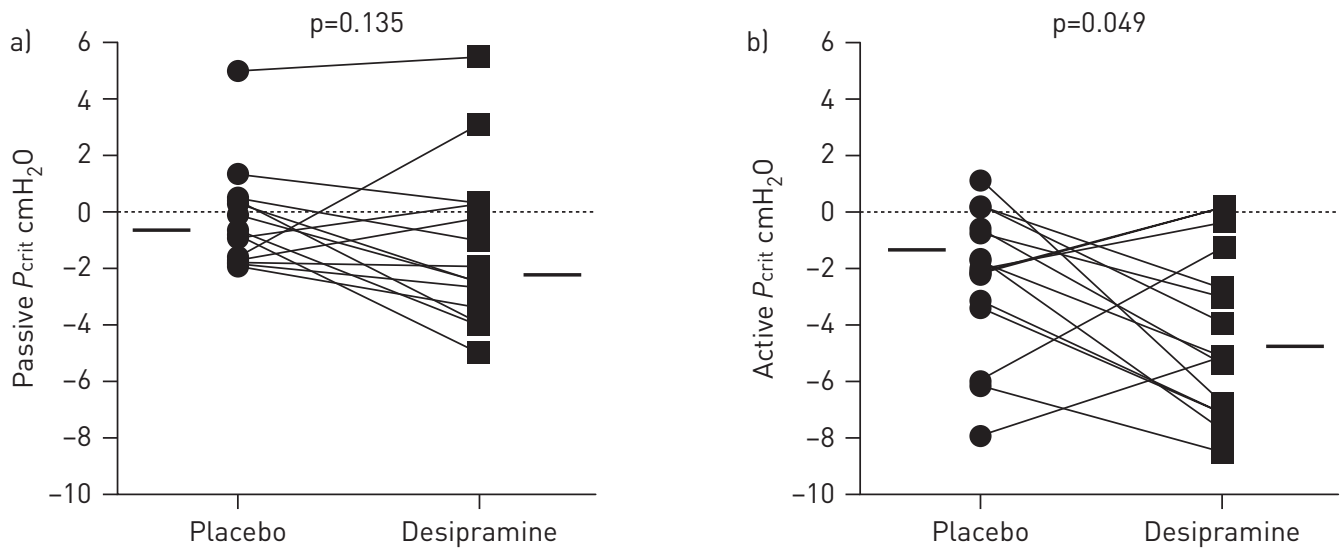

FIGURE 2 Individual data showing the effect of desipramine on the a) passive and b) active critical closing pressure (critical collapsing pressure of the upper airway $\left(P_{\text {crit }}\right)$ ). Desipramine significantly reduced active $P_{\text {crit }}$ by $3.1 \mathrm{cmH}_{2} \mathrm{O}$ (median change) compared with placebo. A smaller nonsignificant reduction in passive $P_{\text {crit }}$ occurred on drug. Horizontal lines indicate median values. 
TABLE 2 Obstructive sleep apnoea severity in 13 patients who slept supine on both study nights

\begin{tabular}{lccc} 
& Placebo & Desipramine & p-value \\
\hline AHI NREM supine events $\cdot \mathbf{h}^{-1}$ sleep & $42.0(30.8)$ & $34.3(54.9)$ & $>0.5$ \\
ArI NREM supine events $\mathbf{h}^{-1}$ sleep & $54.6(50.3)$ & $56.2(35.2)$ & $>0.5$ \\
$\%$ hypopneas & $78(37)$ & $94(16)$ & 0.11 \\
ODI NREM supine events $\cdot \mathbf{h}^{-1}$ sleep & $28(23)$ & $4(48)$ & $>0.5$ \\
Nadir SaO & $90(3.8)$ & $92(3.8)$ & 0.02
\end{tabular}

Data are presented as median (interquartile range), unless otherwise stated. AHI: apnoea-hypopnoea index; NREM: non-rpaid eye movement sleep; Arl: arousal index; ODI: oxygen desaturation index; $\mathrm{SaO}_{2}$ : arterial oxygen saturation. Individual data are shown in the supplementary data. One subject was excluded from this analysis as he could not sleep while supine on the placebo night.

\section{Post hoc exploratory analysis: physiological determinants of response to desipramine in} moderate-to-severe OSA

In a subgroup of 12 subjects with AHI $>15$ events. $\mathrm{h}^{-1}$, we performed an analysis to determine if baseline phenotypic traits could predict the response to desipramine. Most notably, the improvement in NREM AHI between nights correlated with the improvement in muscle compensation ( $V^{\prime} 0$,active $-V^{\prime} 0$,passive) between nights $(r=-0.72, p=0.009$; see online data supplement). Individual patient data demonstrating the effects of desipramine on the OSA traits in this group of patients are shown in figure 4. Only $V^{\prime}$ arousal was significantly increased on desipramine compared with placebo $(\mathrm{p}=0.03)$.

In order to determine the characteristics of those who gained the greatest benefit of desipramine, we defined a responder to therapy as those whom displayed a reduction in AHI $>20$ events $\cdot \mathrm{h}^{-1}$. We chose this threshold because a change in AHI higher than 20 events $\mathrm{h}^{-1}$ is considered generally beyond normal night-to-night variability $[23,24]$ and the improvement is more likely due to the medication effect. Using this criterion, we identified five responders (dashed line in the graphs in figure 4). Responders had a significantly lower muscle compensation at baseline (0.6 (2.5) versus 2.4 (2.7); $\mathrm{p}<0.01)$. Complete data on the remaining physiological determinants of a response to desipramine are available in the online supplement. Univariate and multivariate analyses including all OSA traits measured during the placebo night (table 3), showed that the baseline muscle compensation was the only significant independent correlate of the change in AHI (figure 5).

\section{Arousal threshold}

Noradrenergic stimulation of the central nervous system could increase wakefulness and reduce the ventilatory drive required to trigger an arousal from sleep. We sought to evaluate this by measuring the arousal threshold on and off the drug. We found that there was a significant reduction in the arousal threshold (waking up more easily) during desipramine compared with placebo night $\left(-13.1(8.7) \mathrm{cmH}_{2} \mathrm{O}\right.$ versus $\left.-16.1(9.3) \mathrm{cmH}_{2} \mathrm{O} ; \mathrm{p}=0.002\right)$ and a consistent trend for a reduced difference between $V^{\prime}$ eupnoea and $V^{\prime}$ arousal on desipramine compared with placebo $(\mathrm{p}=0.07$; table 3$)$, meaning that the patients tended to tolerate less reduction in ventilation without having an arousal from sleep on desipramine night (figure 1). Notably, the three subjects who had a worsening of $P$ crit values and NREM AHI on desipramine had a greater reduction in arousal threshold compared with the other patients (34 (28) versus 21 (19) \% baseline; $\mathrm{p}=0.024$ ).

FIGURE 3 Individual data showing apnoea-hypopnea index (AHI) change from placebo to desipramine. Horizontal lines indicate median values. NREM: non-rapid eye movement sleep.

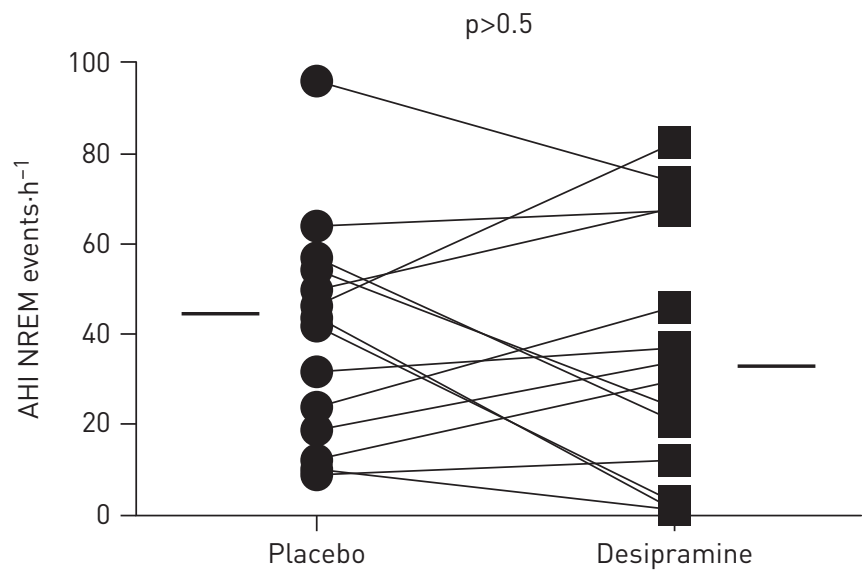


TABLE 3 Physiology parameters during placebo and desipramine night $(n=14)$

\begin{tabular}{|c|c|c|c|}
\hline & Placebo & Desipramine & p-value \\
\hline$V^{\prime}$ eupnoea $L \cdot \min ^{-1}$ & $7.3(1.6)$ & $7(1.3)$ & $>0.5$ \\
\hline$V^{\prime}$ arousal $\mathrm{L} \cdot \mathrm{min}^{-1}$ & $4.8(1.9)$ & $5.4(2.3)$ & 0.1 \\
\hline$V^{\prime} 0$,active $L \cdot \min ^{-1}$ & $2.5(3.2)$ & $4.0(3.4)$ & 0.33 \\
\hline$V^{\prime} 0$,passive $L \cdot \min ^{-1}$ & $0.7(2.6)$ & $1.0(2.0)$ & 0.39 \\
\hline$V^{\prime}$ eupnoea $-V^{\prime}$ arousal $L \cdot \min ^{-1}$ & $2.7(1.4)$ & $1.8(1.7)$ & 0.07 \\
\hline$V^{\prime}$ arousal- $V^{\prime} 0$,active $L \cdot \mathrm{min}^{-1}$ & $2.1(4.4)$ & $0.6(4.2)$ & 0.33 \\
\hline$V^{\prime} 0$,active $-V^{\prime} 0$,passive (muscle compensation) L- $\min ^{-1}$ & $1.5(2.9)$ & $2.4(3.7)$ & 0.24 \\
\hline
\end{tabular}

Data are presented data as median (interquartile range), unless otherwise stated. $V^{\prime}$ eupnoea: eupnoeic ventilation; $V$ 'arousal: ventilation that leads to arousals; $V^{\prime} 0$, passive: ventilation at a nasal pressure of $0 \mathrm{cmH}_{2} \mathrm{O}$ when the pharyngeal muscles are passive; $V_{0}$,active: ventilation at a nasal pressure of $0 \mathrm{cmH}_{2} \mathrm{O}$ when the pharyngeal muscles are active.

\section{Genioglossus responsiveness}

Genioglossus muscle responsiveness to progressively greater epiglottic pressure swings was not different on placebo versus desipramine $\left(-0.25(-0.31)\right.$ versus $\left.-0.24 \quad(-0.33) \% \max \quad \mathrm{cmH}_{2} \mathrm{O} ; \mathrm{p}>0.5\right)$. However, improvement in genioglossus responsiveness with desipramine was associated with improvement in muscle compensation $\left(V^{\prime} 0\right.$,active $-V^{\prime} 0$,passive; $\left.\mathrm{r}=0.74, \mathrm{p}=0.003\right)$ and to the reduction in AHI $(\mathrm{r}=0.73, \mathrm{p}=0.007$; see online data supplement).
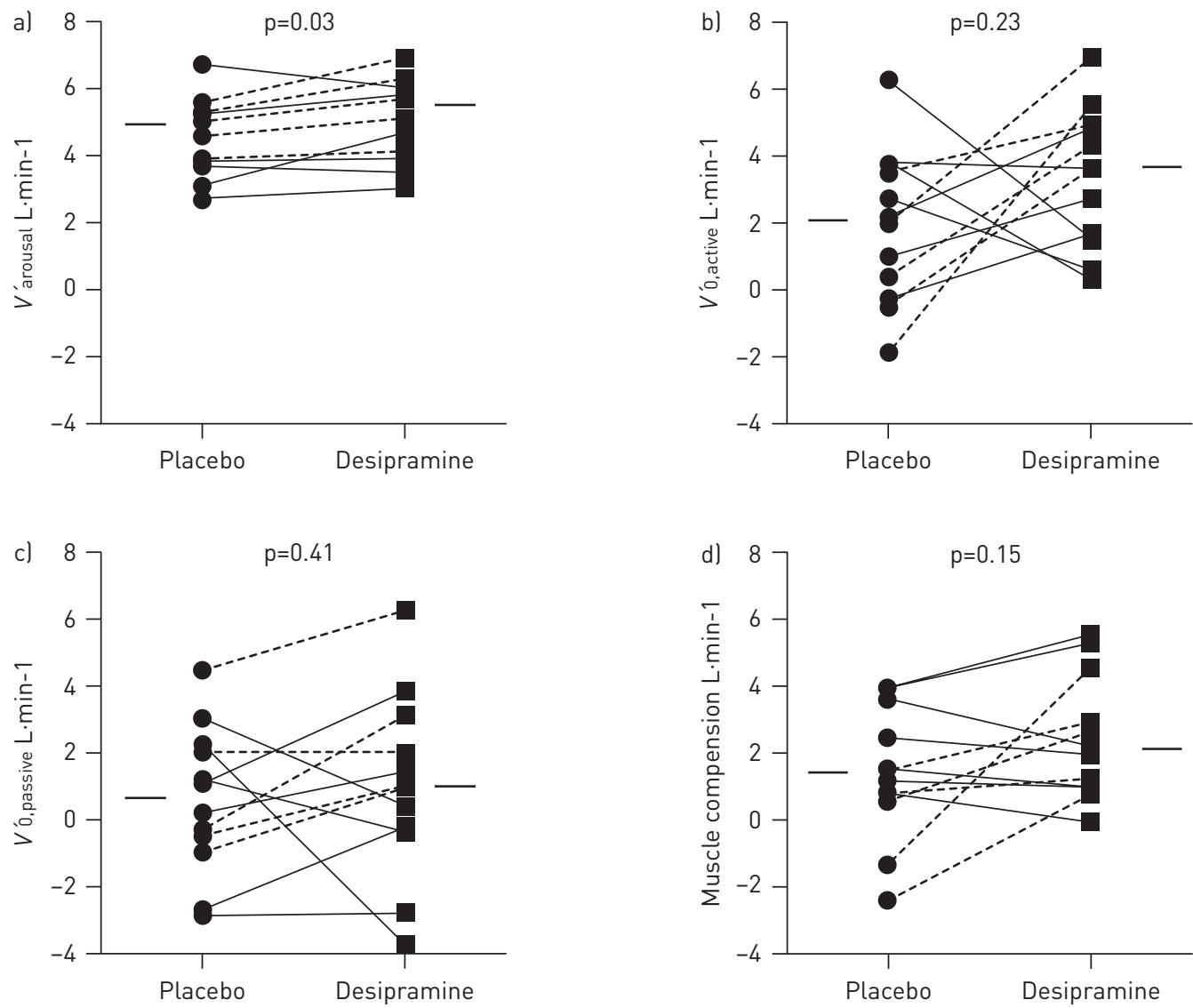

FIGURE 4 Individual data showing changes to al average ventilation of the five stable breaths preceding the arousal if they are flow-limited ( $V$ 'arousal), b) ventilation at atmospheric pressure under maximally active conditions ( $V^{\prime} 0$,active), c) ventilation under eupnoeic conditions $\left(V^{\prime} 0\right.$, passive), and d) muscle compensation $\left(V^{\prime} 0\right.$,active- $V^{\prime} 0$,passive $)$ on placebo and desipramine night in the subgroup of patients with moderate-to-severe obstructive sleep apnoea lapnoea-hypopnoea index (AHI) $>15$ events $\cdot h^{-1}$ ). Responders (AHI reduction $>20$ events $\cdot h^{-1}$ on desipramine compared with placebol are highlighted with a dashed line. 


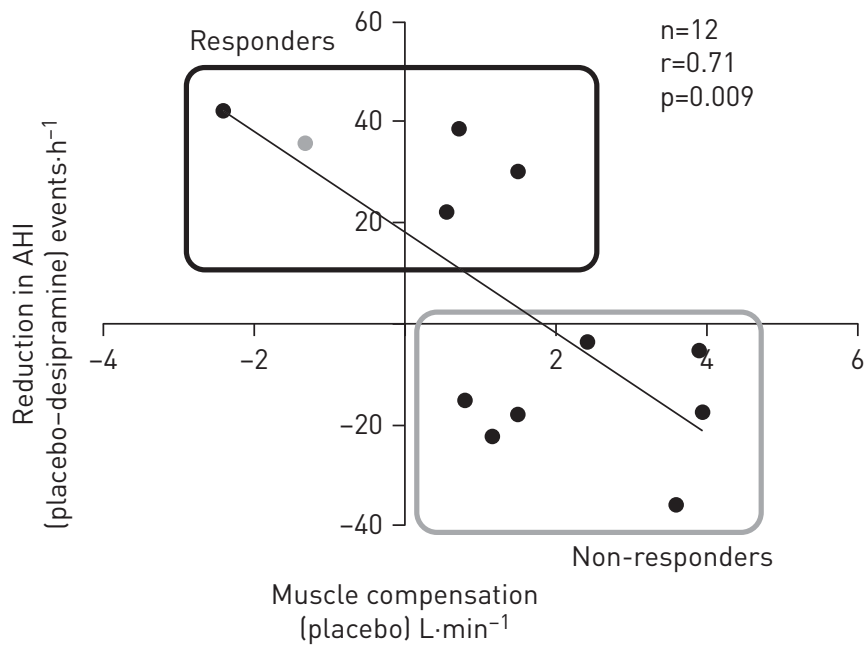

FIGURE 5 Muscle compensation (ventilation at atmospheric pressure under maximally active conditions $\left(V^{\prime} 0\right.$,active $)-v e n t i l a t i o n$ under eupnoeic conditions $\left(V^{\prime} 0\right.$,passive $\left.)\right)$ during placebo night is related to the change in apnoea-hypopnoea index (AHI) from placebo to the desipramine night. Responders had a lower muscle compensation on placebo night $(p<0.01)$ compared with the non-responders. Analysis of change in $\mathrm{AHI}$ was performed on 12 subjects who had $\mathrm{AHI}$ values in the moderate-to-severe range $\left(\geqslant 15\right.$ events. $\left.\mathrm{h}^{-1}\right)$. The grey filled circle indicates that the $\mathrm{AHI}$ value of placebo night was calculated during an additional diagnostic night (see text for explanation). Note that this relationship persisted even when this subject was excluded.

\section{Effects on sleep}

Effects of desipramine on sleep architecture are shown in table 4. NREM stage 1, 2 and 3 were not significantly changed between nights. REM sleep was significantly reduced on desipramine compared with placebo $(\mathrm{p}=0.002)$.

\section{Discussion}

The main finding of this study was that desipramine significantly improves active upper airway collapsibility in patients with OSA. In addition, those who have minimal muscle compensation on placebo show the greatest reduction in AHI on desipramine.

It is noteworthy that active $P_{\text {crit }}$ represents the sum of the passive $P_{\text {crit }}$ and the active neuromuscular response [9]. Although active $P_{\text {crit }}$ was significantly higher on desipramine compared with placebo, the difference between active and passive Pcrit was not significantly different (1.5 (3.0) on placebo versus 2.4 (3.8) $\mathrm{cmH}_{2} \mathrm{O}$ on desipramine; $\mathrm{p}=0.24$ ), suggesting that the observed reduction in the active $P_{\text {crit }}$ on desipramine is likely due to a combination of improvements in passive $P_{\text {crit }}$ and active responses. Interestingly, desipramine was able to reduce collapsibility under active conditions with a median change of $-3.1 \mathrm{cmH}_{2} \mathrm{O}(4.8)$, similar to the change in collapsibility obtained with electrical hypoglossal-nerve stimulation [25] which is an effective therapy in a subgroup of patients [26] that still requires better characterisation [27].

These results confirm, at least in part, what we recently observed in a group of 10 non-apnoeic control subjects [11], for whom administration of desipramine $200 \mathrm{mg}$ by mouth before sleep induced a consistent

\begin{tabular}{|c|c|c|c|}
\hline & Placebo & Desipramine & p-value \\
\hline TST min & $266.0(71.5)$ & 266.5 (124.0) & $>0.5$ \\
\hline SE \% & $60.4(16.5)$ & $62.2(15.4)$ & $>0.5$ \\
\hline NREM1 \%TST & 28.5 (14.7) & $34.0(20.5)$ & 0.110 \\
\hline NREM2 \%TST & $51.0(4.0)$ & $55.4(17.2)$ & 0.058 \\
\hline NREM3 \%TST & $4.5(13.1)$ & $0.9(4.2)$ & 0.175 \\
\hline REM \%TST & $14.5(10.9)$ & $2.0(6.1)$ & 0.002 \\
\hline
\end{tabular}

Data are presented as median (interquartile range), unless otherwise stated. TST: total sleep time; SE: sleep efficiency; NREM1, NREM2 and NREM3: non-rapid eye movement sleep stages 1, 2 and 3; REM: rapid eye movement sleep. 
increase in genioglossal tonic activity and a reduction in passive Pcrit. Here, in OSA patients, we found a similar magnitude non-significant reduction in passive $P$ crit whereas active $P$ crit was significantly reduced. There are several possibilities that may explain these differences. First, the small number of subjects studied for passive $P_{\text {crit }}$ may have led to type II error; to detect a true difference of $1.3 \pm 2.4 \mathrm{cmH}_{2} \mathrm{O}$ that we found in this study with statistical significance if present, 17 more subjects would be required. Secondly, it might be inappropriate to compare passive and active Pcrit in controls versus OSA patients because the ranges of CPAP pressures used in the two studies were different (the median optimum CPAP pressure for controls was 3.5 (4.3) $\mathrm{cmH}_{2} \mathrm{O}$ while for OSA patients it was 8.0 (3.3) $\left.\mathrm{cmH}_{2} \mathrm{O} ; \mathrm{p}<0.01\right)$ and different levels of optimum CPAP could cause different degrees of upper airway muscle relaxation [28] or lung inflation [29], which could affect passive Pcrit measurements. Third, most of the CPAP drops were performed in a sub-atmospheric (negative) pressure range for controls, while pressure drops in OSA were predominantly in a positive pressure range; this difference might lead to different behaviour of upper airway muscles in the two groups as constant intrapharyngeal negative pressure increases EMGGG activity within and between breaths [30, 31].

Although desipramine significantly improved upper airway collapsibility, in the post hoc exploratory analysis, we found that the only phenotype predictor of an AHI reduction was minimal upper airway muscle compensation at baseline. On the contrary, patients with good muscle compensation off drug did not show a reduction in OSA severity despite improvements in collapsibility. In contrast to our hypothesis, the degree of collapsibility at baseline did not predict the outcome on drug in this small group of patients. However, all but one of the patients included in the present study had a mild-to-moderate upper airway anatomical defect. It would be interesting to study other patients with a wider range of $P_{\text {crit value to }}$ confirm that only muscle compensation can predict desipramine outcome.

Three subjects in this study had a worsening of AHI and collapsibility on desipramine. The reason for this observation is not clear. However, one possible explanation might be the reduced arousal threshold with desipramine which could affect both OSA severity and collapsibility. Indeed, these three subjects had a median reduction of arousal threshold of $34 \%$ versus $21 \%(\mathrm{p}=0.024)$ in other patients. It has been shown that a low arousal threshold may contribute to OSA pathogenesis because it does not allow time for an adequate upper airway muscles response [32]. In patients with moderate-to-severe OSA, the only ventilatory trait that was significantly increased on desipramine compared with placebo was the $V^{\prime}$ arousal, consistent with the reduced arousal threshold that we measured with the epiglottic pressure catheter, meaning that the patients could tolerate less ventilatory reduction on desipramine compared with placebo. These observations suggest that desipramine at this dose $(200 \mathrm{mg})$ might have significant wake-promoting activity and that lowering the dose of this drug or combining desipramine with a non-myorelaxing sedative to raise the arousal threshold might be a successful treatment strategy in certain individuals.

Two previous randomised controlled trials $[15,16]$ assessed the effects of the TCA protriptyline on OSA severity. BROwNell et al. [15] found no change in AHI during NREM sleep after 4 weeks of therapy with protriptyline $20 \mathrm{mg}$ in a group of 5 obese men with severe OSA. In another double-blind trial, WhYTE et al. [16] found that administration of protriptyline $20 \mathrm{mg}$ for 14 days in 10 moderate-to-severe OSA patients did not change NREM AHI as a group but generated wide inter-individual variability in the change in AHI. Our study is different from the previously cited ones in many respects. We administered a high dose of desipramine for one night and evaluated the patients' phenotypic traits. We chose desipramine rather than protriptyline because of its potent adrenergic effect compared to the other TCAs [33]. Similar to previous clinical trials testing TCAs $[15,16]$, we did not find systematic changes in AHI with desipramine treatment in unselected patients. However, we did demonstrate a significant improvement in collapsibility with desipramine and the measurement of phenotypic traits allowed us to identify a particular phenotype of OSA patient in whom desipramine therapy was effective, namely those with minimal upper airway compensation at baseline.

\section{Limitations}

This study has several limitations. First, the small number of subjects studied in this trial does not allow for precise conclusions regarding the OSA patient profile of those responding to desipramine, although we were able to generate a working hypothesis to be tested in further studies. Second, given that the AHI determined from our study was compiled from recordings taken both before and after the physiological measurements, our values may be not representative of the true AHI and as such should be interpreted with caution. However, in a recent study, CHOU et al. [34] validated the split-night procedure in which OSA is diagnosed only during half-night polysomnography [35] showing that partial-night polysomnography $(1$ or $2 \mathrm{~h}$ ) can be as useful as whole-night polysomnography for OSA diagnosis, especially for patients with severe disease. We note that the median value of total sleep time off CPAP for both placebo and desipramine nights was over $2 \mathrm{~h}$ in our study.

Despite these considerations, at this time we cannot completely exclude that night-to-night variability of OSA severity, rather than desipramine effect, influenced the change in AHI. Prospective administration of 
desipramine to a selected group of patients with minimal upper airway muscle compensation will answer this question.

Third, the medication effect could also be different in different parts of the night given that desipramine has shown different time to peak concentration between individuals, generally being between 4 to $6 \mathrm{~h}$ [36]. Although plasma concentration of desipramine was not measured in this trial, we believe that this limitation is mitigated by the high affinity of desipramine for norepinephrine transporter, so that a small plasma concentration would be expected to saturate these receptors [33]. Fourth, we measured the effects of desipramine after a single-night administration, the effects of prolonged administration are unknown, but it might further improve upper airway collapsibility. Indeed, in animal studies, chronic administration of desipramine led to downregulation of the norepinephrine transporter on the presynaptic membrane, which would additionally increase the availability of postsynaptic norepinephrine [37].

\section{Conclusions}

In this study, we showed that desipramine reduced upper airway collapsibility when the upper airway was challenged with gradual reduction in airway pressure in OSA patients. In addition, measurement of OSA phenotypes showed that the greatest reductions in AHI occurred in subjects with minimal upper airway response at baseline. Despite these improvements, arousal threshold was reduced on desipramine and this could be the reason for OSA worsening in some patients. These findings offer new insights for the development of pharmacological treatments for OSA and in particular for the identification of a group of OSA subjects who might respond to desipramine treatment.

\section{Acknowledgements}

Author contributions are as follows. L. Taranto-Montemurro contributed to study design, data collection, data analysis and interpretation, and drafting and review of the manuscript for important intellectual content. S.A. Sands contributed to study design, data collection, data analysis and interpretation and review of the manuscript. B.A. Edwards contributed to the study design, data collection and interpretation and review of the manuscript. A. Azarbarzin contributed to data collection, data analysis and review of the manuscript. M. Marques contributed to the data collection and review of the manuscript. C. de Melo contributed to the data collection and review of the manuscript. D.J. Eckert contributed to data interpretation and review of the manuscript. D.P. White contributed to data analysis and interpretation and review of the manuscript for important intellectual content. A. Wellman contributed to the study design, data analysis and interpretation, and drafting and review of the manuscript for important intellectual content.

\section{References}

1 Punjabi NM. The epidemiology of adult obstructive sleep apnea. Proc Am Thorac Soc 2008; 5: $136-143$.

2 White DP, Younes MK. Obstructive sleep apnea. Compr Physiol 2012; 2: 2541-2594.

3 Sawyer AM, Gooneratne NS, Marcus CL, et al. A systematic review of CPAP adherence across age groups: clinical and empiric insights for developing CPAP adherence interventions. Sleep Med Rev 2011; 15: 343-356.

4 Gottlieb DJ, Yenokyan G, Newman AB, et al. Prospective study of obstructive sleep apnea and incident coronary heart disease and heart failure: the sleep heart health study. Circulation 2010; 122: 352-360.

5 Punjabi NM, Caffo BS, Goodwin JL, et al. Sleep-disordered breathing and mortality: a prospective cohort study. PLoS Med 2009; 6: e1000132.

6 Redline S, Yenokyan G, Gottlieb DJ, et al. Obstructive sleep apnea-hypopnea and incident stroke: the sleep heart health study. Am J Respir Crit Care Med 2010; 182: 269-277.

7 Loewen AH, Ostrowski M, Laprairie J, et al. Response of genioglossus muscle to increasing chemical drive in sleeping obstructive apnea patients. Sleep 2011; 34: 1061-1073.

8 Eckert DJ, White DP, Jordan AS, et al. Defining phenotypic causes of obstructive sleep apnea. Identification of novel therapeutic targets. Am J Respir Crit Care Med 2013; 188: 996-1004.

9 McGinley BM, Schwartz AR, Schneider H, et al. Upper airway neuromuscular compensation during sleep is defective in obstructive sleep apnea. J Appl Physiol (1985) 2008; 105: 197-205.

10 Chan E, Steenland HW, Liu H, et al. Endogenous excitatory drive modulating respiratory muscle activity across sleep-wake states. Am J Respir Crit Care Med 2006; 174: 1264-1273.

11 Taranto-Montemurro L, Edwards BA, Sands SA, et al. Desipramine increases genioglossus activity and reduces upper airway collapsibility during non-REM sleep in healthy subjects. Am J Respir Crit Care Med 2016; in press [DOI: 10.1164/rccm.201511-2172OC].

12 Conway WA, Zorick F, Piccione P, et al. Protriptyline in the treatment of sleep apnoea. Thorax 1982; 37: 49-53.

13 Hanzel DA, Proia NG, Hudgel DW. Response of obstructive sleep apnea to fluoxetine and protriptyline. Chest 1991; 100: 416-421.

14 Smith PL, Haponik EF, Allen RP, et al. The effects of protriptyline in sleep-disordered breathing. Am Rev Respir Dis 1983; 127: 8-13.

15 Brownell LG, West P, Sweatman P, et al. Protriptyline in obstructive sleep apnea: a double-blind trial. $N$ Engl J Med 1982; 307: 1037-1042.

16 Whyte KF, Gould GA, Airlie MA, et al. Role of protriptyline and acetazolamide in the sleep apnea/hypopnea syndrome. Sleep 1988; 11: 463-472.

17 Sleep-related breathing disorders in adults: recommendations for syndrome definition and measurement techniques in clinical research. The Report of an American Academy of Sleep Medicine Task Force. Sleep 1999; 22 : 667-689.

18 Jordan AS, White DP, Owens RL, et al. The effect of increased genioglossus activity and end-expiratory lung volume on pharyngeal collapse. J Appl Physiol (1985) 2010; 109: 469-475. 
Sands SA, Eckert DJ, Jordan AS, et al. Enhanced upper-airway muscle responsiveness is a distinct feature of overweight/obese individuals without sleep apnea. Am J Respir Crit Care Med 2014; 190: 930-937.

20 Berry RB, Budhiraja R, Gottlieb DJ, et al. Rules for scoring respiratory events in sleep: update of the 2007 AASM Manual for the Scoring of Sleep and Associated Events. Deliberations of the Sleep Apnea Definitions Task Force of the American Academy of Sleep Medicine. J Clin Sleep Med 2012; 8: 597-619.

21 Wellman A, Edwards BA, Sands SA, et al. A simplified method for determining phenotypic traits in patients with obstructive sleep apnea. J Appl Physiol (1985) 2013; 114: 911-922.

22 Terrill PI, Edwards BA, Nemati S, et al. Quantifying the ventilatory control contribution to sleep apnoea using polysomnography. Eur Respir J 2015; 45: 408-418.

23 Bliwise DL, Benkert RE, Ingham RH. Factors associated with nightly variability in sleep-disordered breathing in the elderly. Chest 1991; 100: 973-976.

24 White LH, Lyons OD, Yadollahi A, et al. Night-to-night variability in obstructive sleep apnea severity: relationship to overnight rostral fluid shift. J Clin Sleep Med 2015; 11: 149-156.

25 Oliven A, O'Hearn DJ, Boudewyns A, et al. Upper airway response to electrical stimulation of the genioglossus in obstructive sleep apnea. J Appl Physiol (1985) 2003; 95: 2023-2029.

26 Strollo PJ, Soose RJ, Maurer JT, et al. Upper-airway stimulation for obstructive sleep apnea. N Engl J Med 2014; 370: 139-149.

27 Malhotra A. Hypoglossal-nerve stimulation for obstructive sleep apnea. N Engl J Med 2014; 370: $170-171$.

28 Deegan PC, Nolan P, Carey M, et al. Effects of positive airway pressure on upper airway dilator muscle activity and ventilatory timing. J Appl Physiol (1985) 1996; 81: 470-479.

29 Stanchina ML, Malhotra A, Fogel RB, et al. The influence of lung volume on pharyngeal mechanics, collapsibility, and genioglossus muscle activation during sleep. Sleep 2003; 26: 851-856.

30 Schwartz AR, O'Donnell CP, Baron J, et al. The hypotonic upper airway in obstructive sleep apnea: role of structures and neuromuscular activity. Am J Respir Crit Care Med 1998; 157: 1051-1057.

31 Malhotra A, Pillar G, Fogel RB, et al. Pharyngeal pressure and flow effects on genioglossus activation in normal subjects. Am J Respir Crit Care Med 2002; 165: 71-77.

32 Eckert DJ, Malhotra A, Wellman A, et al. Trazodone increases the respiratory arousal threshold in patients with obstructive sleep apnea and a low arousal threshold. Sleep 2014; 37: 811-819.

33 Gillman PK. Tricyclic antidepressant pharmacology and therapeutic drug interactions updated. Br J Pharmacol 2007; 151: 737-748

34 Chou K-T, Chang Y-T, Chen Y-M, et al. The minimum period of polysomnography required to confirm a diagnosis of severe obstructive sleep apnoea. Respirology 2011; 16: 1096-1102.

35 Epstein LJ, Kristo D, Strollo PJ Jr, et al. Clinical guideline for the evaluation, management and long-term care of obstructive sleep apnea in adults. J Clin Sleep Med 2009; 5: 263-276.

36 Weiner D, Garteiz D, Cawein M, et al. Pharmacokinetic linearity of desipramine hydrochloride. J Pharm Sci 1981; 70: 1079-1080.

37 Zhao Z, Baros AM, Zhang HT, et al. Norepinephrine transporter regulation mediates the long-term behavioral effects of the antidepressant desipramine. Neuropsychopharmacology 2008; 33: 3190-3200. 